\title{
Tecnología de futuro: cirugía robótica Da Vinci
}

\author{
H. Villavicencio Mavrich \\ Director del Servicio de Urología. Fundació Puigvert. Barcelona
}

Actas Urol Esp 2005; 29 (10): 919-921

$\mathrm{L}^{\mathrm{a}}$ a Urología se encuentra actualmente en un momento histórico de extraordinario desarrollo tecnológico. Un ejemplo claro de este proceso de crecimiento y modernización para el beneficio y satisfacción de los pacientes y de los profesionales de la Urología es la incorporación de la cirugía robótica avanzada Da Vinci.

Esta nueva tecnología se aprobó por la FDA para la Urología en el 2001. Los primeros estudios demostraron la gran viabilidad y seguridad que situaba el futuro de la cirugía urológica a una velocidad multiplicada y a nuestro alcance.

\section{BREVE HISTORIA}

La primera cirugía robótica con éxito se realizó en 1999. En el año 2000 significó la revolución definitiva del cirujano antes llamado "manos de plata" por "manos de acero". En el 2001 se realizó la primera intervención transoceánica, el cirujano estaba en Nueva York (EE.UU.) y el paciente en Estrasburgo (Francia). La intervención fue una colecistectomía y, pese a la distancia, fue un éxito, haciéndose realidad un sueño de la NASA y del Departamento de Estado Americano, de aplicar una tecnología de telecirugía a distancia con la intención de aplicarla en las estaciones espaciales, plataformas petroliferas alejadas de ultramar y en el propio campo de batalla.

La transmisión tenía un retardo de sólo 150 milisegundos (ida y vuelta de la señal), a pesar de existir entre las dos ciudades una distancia de 7.500 kilómetros.

\section{HACIA EL FUTURO}

La incorporación de nuevos avances tecnológicos, como la cirugía robótica, a nivel mundial, abre las puertas a alternativas terapéuticas menos invasivas y más precisas para el paciente, inimaginables hace algunos años.
Si bien la laparoscopia convencional supuso una gran ventaja para el paciente con relación a la cirugía abierta y su paulatina sustitución, la tecnología robótica Da Vinci está dirigida a suplir las limitaciones de la cirugía laparoscópica.

El robot devuelve los grados de libertad perdidos en la laparoscopia convencional, facilita la manipulación de instrumentos articulados muy pequeños y finos en la cavidad abdominal, elimina el temblor de las manos del cirujano y, por consiguiente, las cirugías son más precisas al tener el cirujano una visión tridimensional a través de un visor estéreo de alta resolución de inmersión. Aporta una perfecta alineación coxial ojos-manos que evita el estrés del cirujano, ofreciendo también una posición mucho más ergonómica y a distancia del paciente.

\section{PARA EL PACIENTE}

Todo ello se traduce en beneficios claros para el paciente: cirugía mínimamente invasiva, menos complicaciones, casi ausencias de transfusiones sanguíneas, menos medicación para mitigar el dolor, menos estancia hospitalaria y, sobre todo, brevísima convalecencia, haciendo que los pacientes se incorporen a las actividades normales de forma rápida (calidad de vida).

La primera intervención quirúrgica con el robot Da Vinci realizada en España, ha sido precisamente urológica. Concretamente, el 6 de julio de 2005 realicé la primera prostatectomía radical por cáncer, con una duración de tres horas y media. La número veinte, duró una hora y cuarenta minutos, con excelente satisfacción por parte de los pacientes y para mí como cirujano.

Aunque se pueden hacer todas las cirugias urológicas, está principalmente indicada para procedimientos de alta complejidad y necesidad de disección, como es la prostatectomía radical 
por cáncer, con pelvis estrecha y suturas complejas, permitiendo a su vez una importante preservación de la potencia eréctil y de la continencia urinaria.

\section{SISTEMA Da VINCI}

La fusión de dos empresas con sede en el Silicon Valley, de California (Computer Motion, INC y Da Vinci Surgical System, de Intuitive), dio lugar a la INTUITIVE SURGICAL INC (2003), cuyo producto final es el sistema de telemanipulación robótica Da Vinci, en honor a Leonardo (1452): artista, inventor, descubridor, astrónomo, pintor (La Gioconda), anatomista, ingeniero y militar.

Consta de una consola de control, donde el cirujano se sienta a manipular el robot, a una distancia del paciente.

El cirujano obtiene una visión estereoscópica, que produce una imagen tridimensional con magnificación de hasta 10-15 aumentos del campo operatorio.

Los instrumentos del robot están miniaturizados y son muy finos (de 2-4 mm), con una excelente articulación distal que permite siete grados de libertad de movimientos (Fig. 1).

El cirujano acciona los controles en la consola y los brazos del robot reproducen fielmente y con precisión absoluta los movimientos del operador, en el campo quirúrgico a tiempo real, no existe retraso entre los movimientos del cirujano y el movimiento de los instrumentos del robot. Además de la precisión, tiene una ventaja sobreañadida: el procesador elimina cualquier temblor o movimiento innecesario del cirujano (Fig. 2).

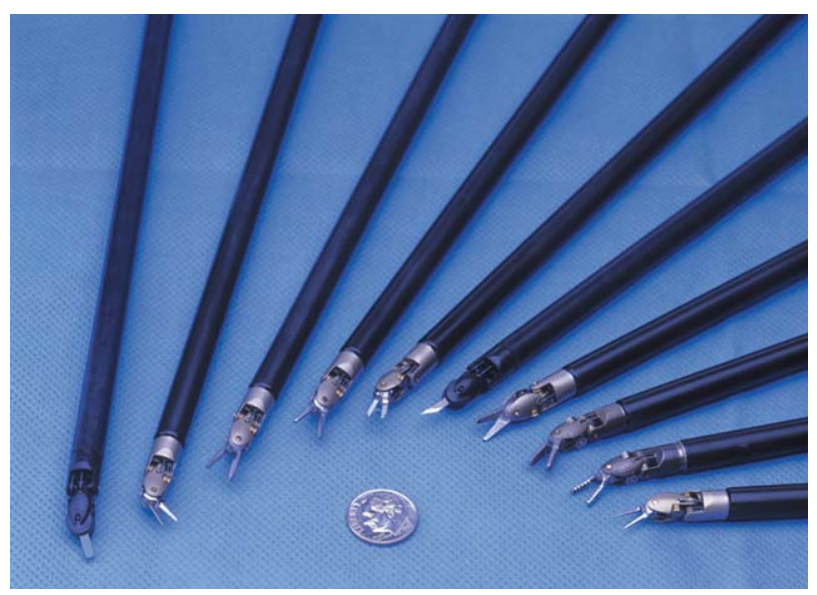

FIGURA 1

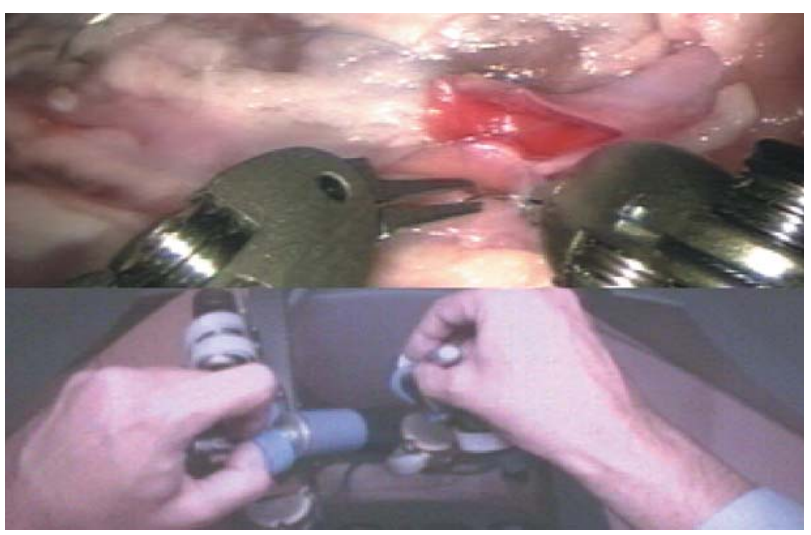

FIGURA 2

El robot dispone de tres brazos quirúrgicos articulados y los más modernos, como el nuestro, disponen de 4 brazos: dos quirúrgicos y uno central que porta las luces y cámara de vídeo, con dos imágenes de doble canal, que se funden y dan una imagen estereoscópica tridimensional. El cuarto brazo hace de ayudante traccionadorseparador. Completa el robot, una torre de monitorización y visualización para el aprendizaje a tiempo real del ayudante y visitantes.

\section{TECNOLOGÍA PUNTA}

Para cirujanos experimentados que pueden reproducir fielmente la cirugía abierta con una tecnología de vanguardia y curva de aprendizaje relativamente corta.

La cirugía robótica sólo exige experiencia en cirugía abierta, operar dos días en cadáveres (La Sorbona, de París (Francia) es la central para Europa) y supervisión de las primeras intervenciones por un experto.

En el año 2000 se habían realizado 36 cirugías urológicas de prueba y en el 2001, la FDA aprobó el robot como tecnología quirúrgica urológica dadas sus altas prestaciones. En el 2004 se practicaron unas 8.400 prostatectomías, para este año 2005, está previsto llegar a las 16.500 y la proyección esperada para el 2006 ronda las 33.500 (Figs. 3 y 4). Además de la cirugía urológica, la FDA ha aprobado también la cirugía robótica para cirugía cardíaca, torácica, pediátrica, cirugía general y ginecológica.

Es una tecnología que va a más y sin retorno. La cirugía laparoscópica es una tecnología en 


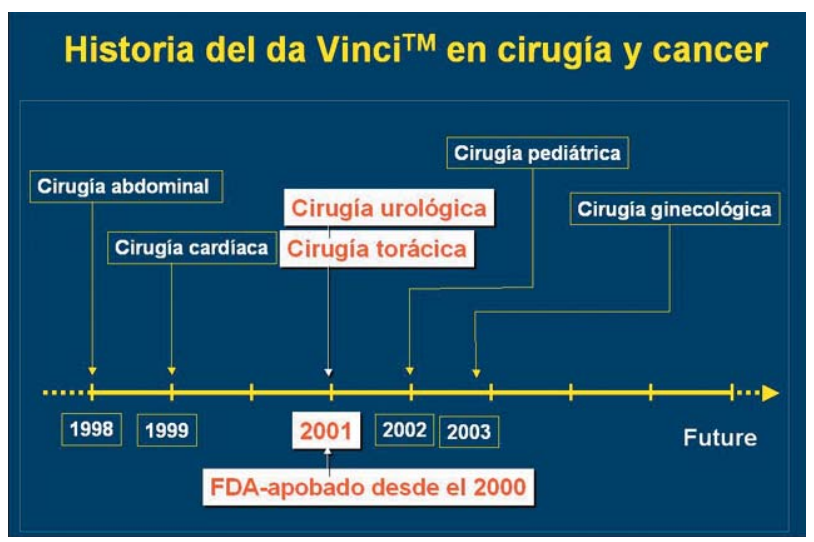

FIGURA 3

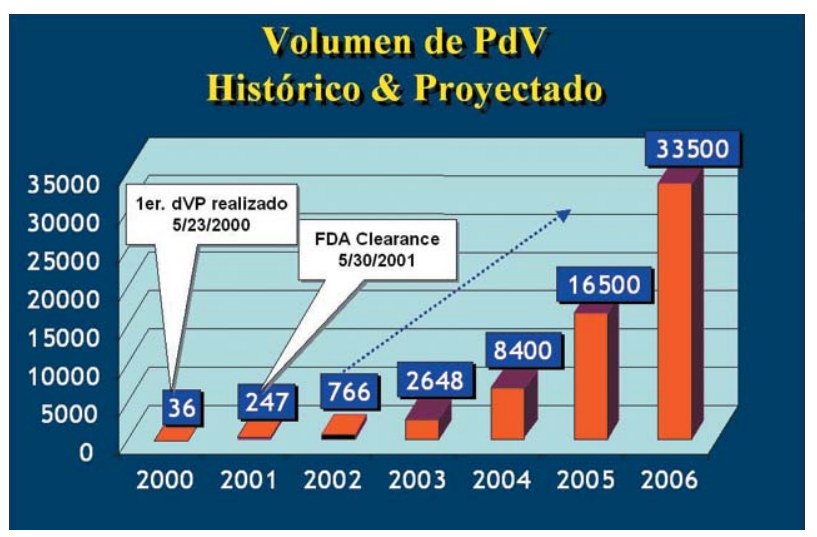

\section{FIGURA 4}

proceso de transición hacia la cirugía robótica. La calidad de la imagen tridimensional intraoperatoria con sensación de profundidad, la perfecta sincronización manos-ojos, la precisión de los instrumentos, la exactitud de sus suturas y la exéresis que se realiza con mayor destreza y confort, hará que pronto la cirugía robótica sea ampliamente difundida y realizada de forma rutinaria en el mundo entero.

\section{REFERENCIAS}

Menon M, Shrivastava A, Tewari A. Laparoscopic radical prostatectomy: conventional and robotic. Urology 2005 nov: 66 (5 suppl):101-104.

Tewari A, Kaul S, Menon M. Robotic radical prostatectomy: a minimally invasive terapy for prostate cancer. Curr Urol Rep 2005 feb;6(1):45-48.
Menon M, Hemal AK, VIP Team. Vattikuti Institute prostatectomy: a technique of robotic radical prostatectomy; experience in more than 1000 cases. J Endourol 2004 sep;18(7):611-619. Menon M, Hemal AK, Tewari A, Shrivastava A, Bhandari A. The technique of apical dissection of the prostate and urethrovesical anastomosis in robotic radical prostatectomy. BJU Int 2004 apr;93(6):715-719.

Hemal AK, Menon M. Robotics in Urology. Curr Opin Urol 2004 mar; 14(2):89-93. Review.

Menon M, Hemal AK, Tewari A, Shrivastava A, Shoma AM, ElTabey NA, Shaaban A, Abol-Eneim H, Ghoneim MA. Nervesparing robot-assisted radical cystoprostatectomy and urinary diversion. BJU Int 2003 aug;92(3):232-236.

Tewari A, Srivasatava A, Menon M, Members of the VIP Team. A prospective comparison of radical retropubic and robotassisted prostatectomy: experience in one institution. BJU Int 2003 aug;92(3):205-210.

Tewari A, Peabody JO, Fischer M, Sarle R, Vallencien G, Delmas V, Hassan M, Bansal A, Hemal AK, Guillonneau B, Menon M. An operative and anatomic study to help in nerve sparing during laparoscopic and robotic radical prostatectomy. Eur Urol 2003 may;43(5):444-454.

Menon M. Robotic radical retropubic prostatectomy. BJU Int 2003 feb;91(3): 175-176.

Shah NL, Hemal AK, Menon M. Robot-assisted radical cystectomy and urinary diversion. Curr Urol Rep 2005 mar;6(2):122125.

Hemal AK, Abol-Enein H, Tewari A, Shrivastava A, Shoma AM, Ghoneim MA, Menon M. Robotic radical cystectomy and urinary diversion in the management of bladder cancer. Urol Clin North Am 2004 nov;31(4):719-729.

Hemal AK, Eun D, Tewari A, Menon M. Nuances in the optimum placement of ports in pelvic and upper urinary tract surgery using the Da Vinci robot. Urol Clin North Am 2004 nov;31(4):683-692.

Menon M, Hemal AK, Tewari A, Shrivastava A, Shoma AM, Abol-Ein H. Ghoneim MA. Robot-assisted radical cystectomy and urinary diversion in female patients: technique with preservation of the uterus and vagina. J Am Coll Surg 2004 mar; 198(3):386-393.

Phillips CK, Taneja SS, Stifelman MD. Robot-assisted laparoscopic partial nephrectomy: the NYU technique. J Endourol 2005 may; 19(4):441-445.

Binder J, Brautigam R, Jonas D, Bentas W. Robotic surgery in urology: fact or fantasy?. BJU Int 2004 nov;94(8):1183-1187.
Dr. H. Villavicencio Mavrich
Servicio de Urología
Fundació Puigvert
Cartagena, 340-350
08025 Barcelona 